\title{
Angiopoietin-like protein 2 is a potential biomarker for gastric cancer
}

\author{
TAKUMA YOSHINAGA ${ }^{1,2}$, TAKAMASA SHIGEMITSU ${ }^{1}$, HIROTO NISHIMATA $^{3}$, \\ TAKAYUKI TAKEI $^{1}$ and MASAHIRO YOSHIDA ${ }^{1}$ \\ ${ }^{1}$ Department of Chemical Engineering, Graduate School of Science and Engineering, Kagoshima University, \\ Kagoshima 890-0065; ${ }^{2}$ Division of Clinical Application; ${ }^{3}$ Department of \\ Gastroenterology, Nanpuh Hospital, Kagoshima 892-0854, Japan
}

Received December 17, 2013; Accepted September 18, 2014

DOI: $10.3892 / \mathrm{mmr} .2014 .3040$

\begin{abstract}
Globally, gastric cancer is one of the most common types of cancer and is the second leading cause of cancer-induced mortality. Early detection of gastric cancer is able to contribute to a reduction of its mortality. For early detection, more specific and sensitive biomarkers than the classic biomarkers, including carcinoembryonic antigen, carbohydrate antigen 19-9 and C-reactive protein, are required. The present study focused on the evaluation of the potential of angiopoietin-like protein 2 (ANGPTL2) as a novel biomarker for gastric cancer. The expression levels of ANGPTL2 in undifferentiated and differentiated gastric cancer cell lines (HGC-27 and MKN7, respectively) were therefore investigated. Additionally, ANGPTL2 levels in the serum of gastric cancer patients were compared with those of healthy individuals to evaluate the possibility of the protein as a predictive biomarker for gastric cancer. It was established that the expression levels of ANGPTL2 mRNA and protein were higher in undifferentiated HGC-27 cells than those in differentiated MKN7 cells. In a patient study, it was indicated that the levels of ANGPTL2 in the serum of gastric cancer patients were higher than those in healthy controls. The diagnostic performance of ANGPTL2 was assessed by constructing a receiver operating characteristic (ROC) curve and was evaluated by calculating the area under each ROC curve (AUC). For the discrimination of patients with gastric cancer from healthy individuals, the AUC for ANGPTL2 was $0.774(\mathrm{P}=0.005)(95 \%$ confidence interval, 0.615-0.933). These results suggested that ANGPTL2 was a potential biomarker for gastric cancer.
\end{abstract}

Correspondence to: Professor Masahiro Yoshida, Department of Chemical Engineering, Graduate School of Science and Engineering, Kagoshima University, 1-21-40 Korimoto, Kagoshima 890-0065, Japan

E-mail: myoshida@cen.kagoshima-u.ac.jp

Key words: angiopoietin-like protein 2, gastric cancer, biomarker, serum, HGC-27 cell line, MKN7 cell line

\section{Introduction}

Gastric cancer is one of the most common types of cancer and is the second leading cause of cancer-induced mortality ( $~ 800,000$ mortalities annually) throughout the world (1). Detection of gastric cancer at an early stage markedly reduces its mortality (2). Although certain diagnostic modalities, including endoscopy, have been used to implement early detection, less invasive and more accurate methods are required (3).

Measurement of tumor biomarkers in serum is an alternative screening method for the detection of gastric cancer. To facilitate early detection, more specific and sensitive biomarkers than the classic biomarkers, including carcinoembryonic antigen (CEA), carbohydrate antigen 19-9 (CA19-9) and C-reactive protein (CRP), are required $(1,4-6)$.

ANGPTL2 is a secreted protein belonging to the angiopoietin-like protein family, which regulates angiogenesis $(7,8)$. Angiogenic factors produced by tumor cells are essential for tumor growth (9). A study by Endo et al (10) indicated that ANGPTL2 may be a biomarker for predicting human lung and breast cancers. These previous studies suggested that ANGPTL2 has potential as a biomarker for a variety of cancers in different tissues. To the best of our knowledge, the present study was the first to have evaluated the potential of angiopoietin-like protein 2 (ANGPTL2) as a novel biomarker for gastric cancer.

In order to evaluate the potential of ANGPTL2 as a biomarker for gastric cancer, expression levels of ANGPTL2 in undifferentiated and differentiated gastric cancer cell lines (HGC-27 and MKN7, respectively) were investigated. Additionally, ANGPTL2 levels in serum of gastric cancer patients were compared with those of healthy individuals to investigate the possibility of the protein as a predictive biomarker for gastric cancer.

\section{Materials and methods}

Cell lines. HGC-27, an undifferentiated human gastric cancer cell line and MKN7, a differentiated human gastric cancer cell line, were purchased from RIKEN Cell Bank (Tsukuba, Japan). HGC-27 and MKN7 cells were cultured in Eagle's 
Table I. Characteristics of experimental subjects.

\begin{tabular}{|c|c|c|c|}
\hline Characteristic & Gastric cancer patients $(n=12)$ & Healthy controls $(n=38)$ & Total $(n=50)$ \\
\hline \multicolumn{4}{|l|}{ Age (years) } \\
\hline Mean \pm SD & $66.9 \pm 11.9$ & $47.3 \pm 9.9$ & $52.0 \pm 13.3$ \\
\hline Range & 43-88 & $35-75$ & $35-88$ \\
\hline \multicolumn{4}{|l|}{ Gender } \\
\hline Male & 11 & 22 & 33 \\
\hline Female & 1 & 16 & 17 \\
\hline \multicolumn{4}{|l|}{ BMI $\left(\mathrm{kg} / \mathrm{m}^{2}\right)$} \\
\hline Mean \pm SD & $24.0 \pm 4.5$ & $22.0 \pm 2.8$ & $22.5 \pm 3.3$ \\
\hline Range & $17.9-31.9$ & $17.4-29.5$ & $17.4-31.9$ \\
\hline \multicolumn{4}{|l|}{ Tumor stage } \\
\hline I & 9 & - & 9 \\
\hline II & 3 & - & 3 \\
\hline \multicolumn{4}{|c|}{ Wall infiltration } \\
\hline $\mathrm{M}, \mathrm{SM}$ & 8 & - & 8 \\
\hline MP & 3 & - & 3 \\
\hline SS & 1 & - & 1 \\
\hline
\end{tabular}

SD, standard deviation; BMI, body mass index; M, mucosa; SM, submucosa; MP, tunica muscularis procia; SS, subserosa.

minimum essential medium (Sigma-Aldrich, Gillingham, UK) and RPMI-1640 medium (Sigma-Aldrich), respectively, supplemented with $10 \%$ fetal bovine serum (Life Technologies, Carlsbad, CA, USA), $100 \mathrm{U} / \mathrm{ml}$ penicillin and $100 \mu \mathrm{g} / \mathrm{ml}$ streptomycin (both Meiji Seika Pharma Co., Ltd., Tokyo, Japan) at $37^{\circ} \mathrm{C}$ in $5 \% \mathrm{CO}_{2}$.

Cell culture. HGC-27 and MKN7 cell lines were seeded in six-well plates $\left(6 \times 10^{5}\right.$ cells/well) and incubated at $37^{\circ} \mathrm{C}$ in $5 \%$ $\mathrm{CO}_{2}$. Media in the wells were changed daily. The medium samples were stored at $-80^{\circ} \mathrm{C}$. Cells were collected from the wells daily by trypsinization (Wako Pure Chemical Industries, Ltd., Osaka, Japan) and the cell numbers were determined using a hemocytometer.

Patient samples. The study design was approved by the Ethics Committee of Nanpuh Hospital Kagoshima Kyosaikai, Public Interest Corporation, Japan. Clinical examinations were performed according to the principles in the Declaration of Helsinki. Serum samples were obtained from 50 participants from January to September 2013 at Nanpuh Hospital (Kagoshima, Japan). The participants included 12 patients with gastric cancer [aged 66.9 \pm 11.9 years, mean \pm standard deviation (SD)] and 38 with normal mucosa (aged 47.3 \pm 9.9 years). The histological subtype of the 12 patients with gastric cancer was adenocarcinoma. Nine of the 12 patients with gastric cancer were diagnosed with clinical stage I disease and three were diagnosed with clinical stage II disease. The staging of gastric cancer was based on a routine histopathological analysis and clinical assessment, according to tumor-nodulus-metastases classification. Tumors were classified by the 5th International Union Against Cancer (11). The characteristics of the subjects are summarized in Table I. Informed consent was obtained from all participants.

Measurement of biomarkers in cell culture media and serum. The concentrations of ANGPTL2 in human serum and cell culture medium samples (collected on the second day of cell culture) were determined using an ANGPTL2 ELISA kit (Immuno-Biological Laboratories Co., Ltd., Gunma, Japan). Serum concentrations of CRP were determined by latex agglutination using BM6050 (Kyowa-Medex Co., Ltd., Tokyo, Japan) according to the manufacturer's instructions. Concentrations of CEA and CA19-9 in serum were determined using the electro-chemiluminescence immunoassay using LUMIPULSE G1200 ${ }^{\circledR}$ (Fujirebio, Inc., Tokyo, Japan) according to the manufacturer's instructions. A multiplex suspension array (Bio-Plex Human Angiogenesis 9-Plex Panel; Bio-Rad Laboratories, Hercules, CA, USA) was used to measure the concentrations of the following angiogenic factors in the cell culture medium samples: Granulocyte colony stimulating factor (G-CSF), platelet endothelial cell adhesion molecule-1 (PECAM-1), hepatocyte growth factor (HGF), vascular endothelial growth factor (VEGF), leptin, platelet-derived growth factor-BB (PDGF-BB), angiopoietin-2 and follistatin.

Reverse transcription quantitative polymerase chain reaction (RT-qPCR). HGC-27 and MKN7 cells (5.85x105 cells) were collected from cell culture dishes by trypsinization and the total RNA was extracted from the cells using the High Pure RNA Tissue kit (Roche Diagnostics, Penzberg, Germany) according to the manufacturer's instructions.

The reverse transcription reaction was performed using random hexamer primers (Thermo Fisher Scientific, 


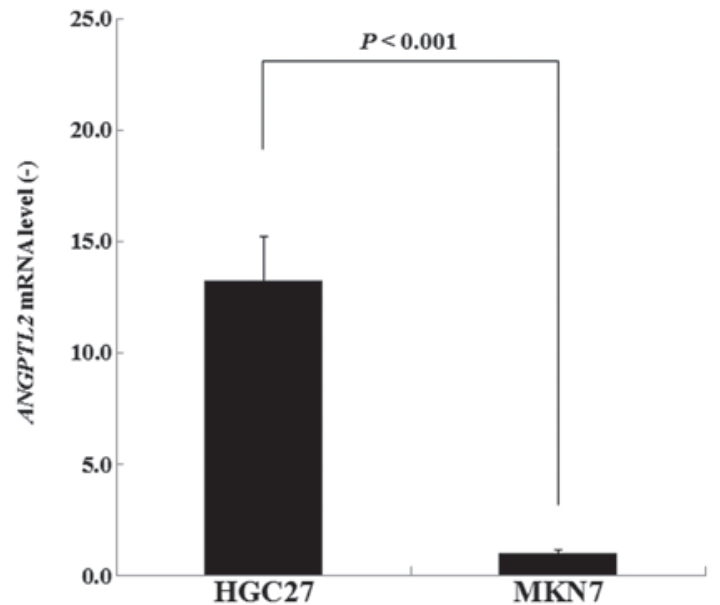

Figure 1. ANGPTL2 mRNA expression of HGC-27 and MKN7 cells ( $\mathrm{n}=6$ ). The level of ANGPTL2 mRNA of HGC-27 cells was significantly higher than that of MKN7 cells. Data are presented as the mean \pm standard deviation. ANGPTL2, angiopoietin-like protein 2; mRNA, messenger RNA.

Waltham, MA, USA) and ReverTra Ace ${ }^{\circledR}$ (Toyobo Co., Ltd., Osaka, Japan) according to the manufacturer's instructions, under the condition that the amount of RNA used was fixed at $200 \mathrm{ng}$.

The amplification of TRAIL variants was performed using the StepOnePlus ${ }^{\mathrm{TN}}$ Real-Time PCR System (Applied Biosciences Life Technologies, Foster City,CA, USA) using the SYBR Green qPCR Mix kit (Toyobo Co., Ltd.) according to the manufacturer's instructions. The specific primers for human ANGPTL2 were: Forward, 5'-GCCACCA AGTGTCAGCCTCA-3' and reverse, 5'-TGGTTCTGAACTGCATTCTGCTG-3' (Life Technologies). Human $\beta$-actin, used as a control, was amplified using the following specific primers: Forward, 5'-TGGCACCCAGCACAATGAA-3' and reverse, 5'-CTA AGTCATAGTCCGCCTAGAAGCA-3' (Life Technologies, Carlsbad, CA, USA) (12).

Statistical analysis. Data were analyzed using SPSS version 14.0J (SPSS, Inc., Chicago, IL, USA) The correlation between the serum ANGPTL2 concentration and different variables was analyzed using Pearson's correlation analysis. The statistical difference between ANGPTL2 concentrations in serum of gastric cancer patients and healthy individuals was analyzed using the rank nonparametric statistical Mann-Whitney $\mathrm{U}$ test. The statistical difference between two groups was analyzed using the Student's t-test. Values are presented as the mean $\pm \mathrm{SD}$. A receiver operating characteristic (ROC) curve was established to evaluate the diagnostic value for differentiating between gastric cancer patients and healthy individuals. $\mathrm{P}<0.05$ was considered to indicate a statistically significant difference between values.

\section{Results}

ANGPTL2 mRNA expression levels are higher in HGC-27 cells than those in MKN7 cells. ANGPTL2 mRNA expression levels of the HGC-27 and MKN7 cells were compared using RT-qPCR. The ANGPTL2 expression levels of HGC-27 were 13.2-fold greater than those of

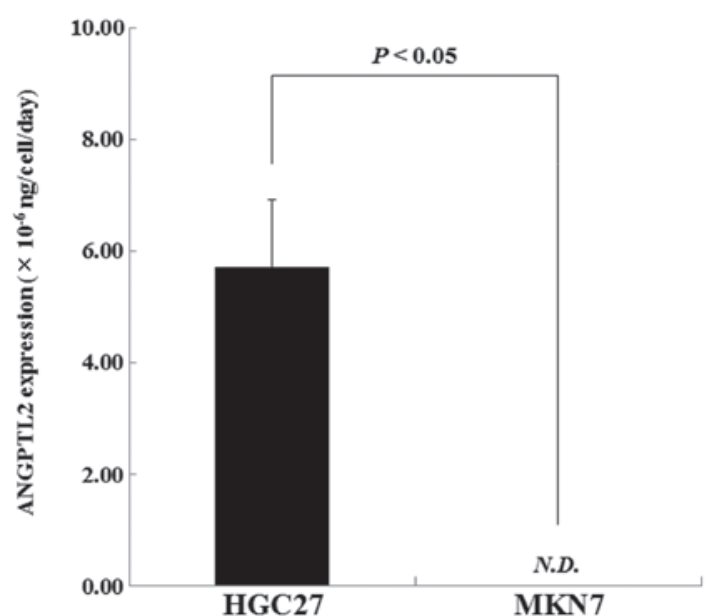

Figure 2. ANGPTL2 expression rate of HGC-27 and MKN7 cells at day two of cell culture $(n=3)$. ANGPTL2 expression was significantly greater in HGC-27 cells than in MKN7 cells. Data are presented as the mean \pm standard deviation. N.D., ANGPTL2 concentrations in cell culture media were lower than the detection limit of ELISA kit (0.05 ng/ml); ANGPTL2, angiopoietinlike protein 2 .

MKN7 (P<0.001; Fig. 1). These results demonstrated that the expression of ANGPTL2 mRNA was significantly higher in undifferentiated HGC-27 cells than in differentiated MKN7 cells.

ANGPTL2 expression rate is greater in HGC-27 cells than that in MKN7 cells. Whether there was a difference in the expression rate of ANGPTL2 between HGC-27 and MKN7 cells was investigated using ELISA. Fig. 2 exhibits the ANGPTL2 expression rate of the cell lines at day two of cell culture. The rate of HGC-27-cell ANGPTL2 production was $5.72 \times 10^{-6} \pm 1.20 \times 10^{-6} \mathrm{ng} / \mathrm{cell} / \mathrm{day}$. The rate of ANGPTL2 production was significantly lower in MKN7 cells than that of HGC-27 cells $(\mathrm{P}<0.05)$. These results demonstrated that the production of ANGPTL2 was considerably higher in undifferentiated HGC-27 cells than in differentiated MKN7 cells.

VEGF is expressed in HGC-27 and MKN7 cells. The expression of angiogenic factors that were potential biomarkers for gastric cancer, including G-CSF, PECAM-1, HGF, VEGF, leptin, PDGF-BB, angiopoietin-2 and follistatin was investigated. Only VEGF expression was confirmed in both cell lines, indicating that VEGF may be a biomarker for gastric cancer (Fig. 3).

ANGPTL2 serum expression levels are increased in patients with gastric cancer. As described above, increased expression levels of ANGPTL2 were confirmed in the HGC-27 cell line in comparison with those in the MKN7 cell line. It was therefore examined whether ANGPTL2 expression was increased in patients with gastric cancers. The concentration of ANGPTL2 in the serum of gastric cancer patients $(4.59 \pm 2.91 \mathrm{ng} / \mathrm{ml})$ was significantly higher than that of healthy individuals $(2.68 \pm 0.60 \mathrm{ng} / \mathrm{ml}, \mathrm{P}<0.01$; Fig. 4$)$. Table II exhibits correlations between serum ANGPTL2 concentration and numerous variables. The ANGPTL2 concentration in the serum of gastric cancer patients demonstrated no significant correlation with 


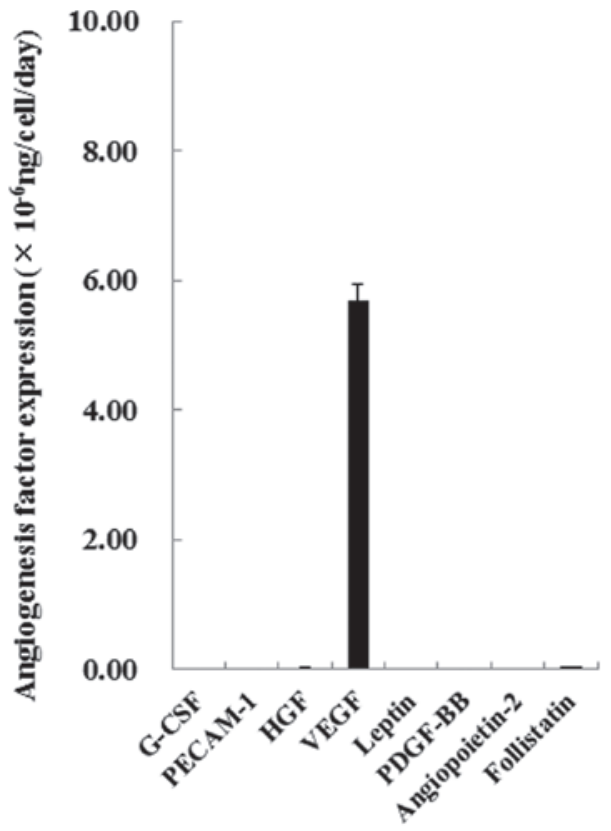

HGC-27

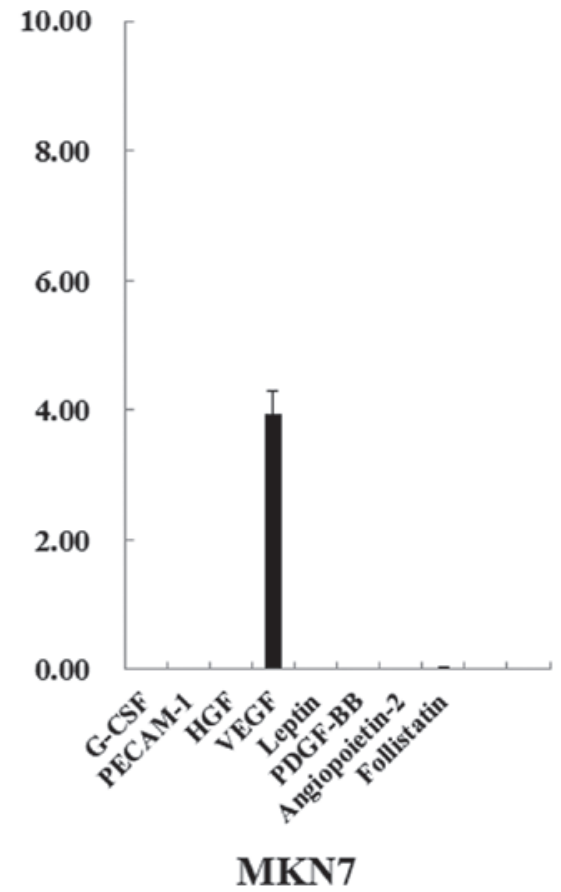

Figure 3. Expression rates of G-CSF, PECAM-1, HGF, VEGF, leptin, PDGF-BB, angiopoietin-2 and follistatin in HGC-27 and MKN7 cells at day two of cell culture $(n=3)$. Data are presented as the mean \pm standard deviation. G-CSF, granulocyte colony stimulating factor; PECAM-1, platelet endothelial cell adhesion molecule-1; HGF, hepatocyte growth factor; VEGF, vascular endothelial growth factor; PDGF-BB, platelet derived growth factor-BB.

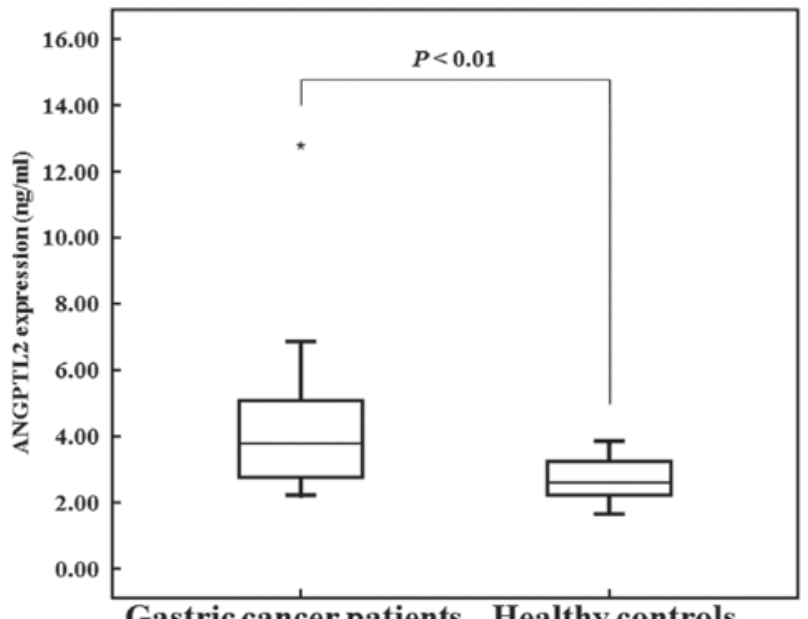

Gastric cancer patients Healthy controls

Figure 4. ANGPTL2 concentration in the serum of gastric cancer patients $(\mathrm{n}=12)$ and healthy controls $(\mathrm{n}=38)$. ANGPTL2 levels were significantly higher in the gastric patients than those in the healthy controls. ANGPTL2, angiopoietin-like protein 2 .

age $(r=-0.323, P=0.307)$, BMI $(r=0.541, P=0.070)$, serum CRP concentration $(\mathrm{r}=0.178, \mathrm{P}=0.579)$, serum $\mathrm{CEA}$ concentration $(\mathrm{r}=-0.083, \mathrm{P}=0.798)$ or $\mathrm{CA} 19-9$ concentration $(\mathrm{r}=-0.312$, $\mathrm{P}=0.324)$. The ANGPTL2 concentration in the serum of the healthy controls also indicated no significant correlation with age $(\mathrm{r}=0.302, \mathrm{P}=0.065), \mathrm{BMI}(\mathrm{r}=0.233, \mathrm{P}=0.160)$, serum $\mathrm{CRP}$ concentration $(\mathrm{r}=0.123, \mathrm{P}=0.463)$, serum $\mathrm{CEA}$ concentration $(\mathrm{r}=0.050, \mathrm{P}=0.767)$ or $\mathrm{CA} 19-9$ concentration $(\mathrm{r}=0.193$, $\mathrm{P}=0.246)$. These data indicated that ANGPTL2 levels in the serum were not significantly associated with these factors and therefore depended solely on the existence of gastric cancer.

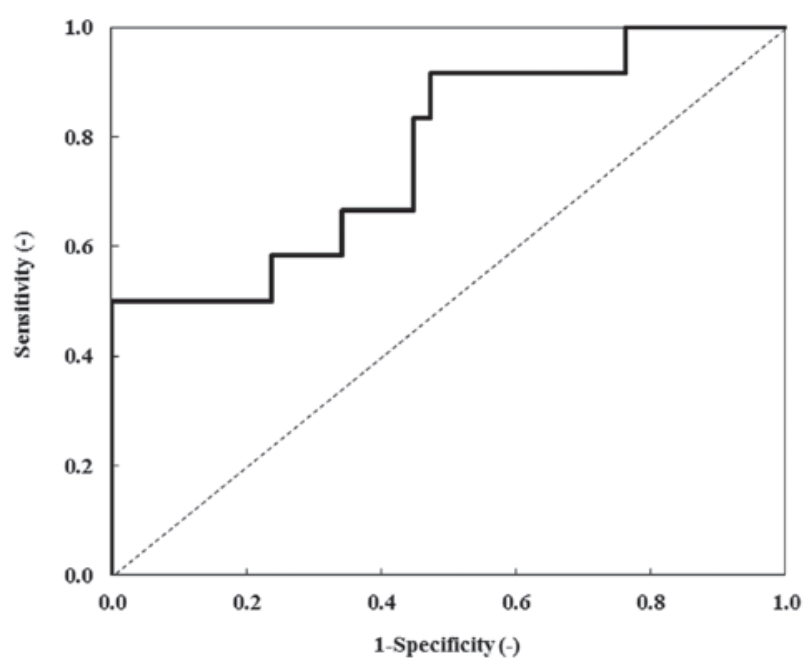

Figure 5. Receiver operating characteristic curve for angiopoietin-like protein 2.

Fig. 5 exhibits the ROC curve for ANGPTL2. Table III indicates the AUC of the ROC curves, in order to evaluate the diagnostic significance of serum ANGPTL2, CRP, CEA and CA19-9 levels of gastric cancer patients. In order to discriminate gastric cancer patients from healthy individuals, the AUC for ANGPTL2 was $0.774[\mathrm{P}=0.005 ; 95 \%$ confidence interval (CI), 0.615-0.933], the AUC for CRP was $0.669(\mathrm{P}=0.080 ; 95 \%$ CI, 0.484-0.853), the AUC for CEA was $0.529(\mathrm{P}=0.768 ; 95 \%$ CI, 0.326-0.731) and the AUC for CA19-9 was $0.570(\mathrm{P}=0.467$; 95\% CI, 0.396-0.744). Thus, the AUC for ANGPTL2 was higher than that of CRP, CEA and CA19-9. These data indicated that ANGPTL2 was a potential biomarker for gastric cancer. 
Table II. Correlations between ANGPTL2 concentration in serum and numerous variables.

\begin{tabular}{|c|c|c|}
\hline \multicolumn{3}{|c|}{ Gastric cancer patients $(n=12)$} \\
\hline Age & -0.323 & 0.307 \\
\hline BMI & 0.541 & 0.070 \\
\hline CRP & 0.178 & 0.579 \\
\hline CEA & -0.083 & 0.798 \\
\hline CA19-9 & -0.312 & 0.324 \\
\hline \multicolumn{3}{|c|}{ Healthy controls $(n=38)$} \\
\hline Age & 0.302 & 0.065 \\
\hline BMI & 0.233 & 0.160 \\
\hline CRP & 0.123 & 0.463 \\
\hline CEA & 0.050 & 0.767 \\
\hline CA19-9 & 0.193 & 0.246 \\
\hline
\end{tabular}

ANGPTL2, angiopoietin-like protein 2; BMI, body mass index; CRP, C-reactive protein; CEA, carcinoembryonic antigen; CA19-9, carbohydrate antigen 19-9.

Table III. AUC for ANGPTL2, CRP, CEA and CA19-9 (gastric cancer patients vs. healthy control).

\begin{tabular}{|c|c|c|c|c|}
\hline Biomarker & AUC & Standard error & P-value & $95 \%$ confidence interval \\
\hline ANGPTL2 & 0.774 & 0.081 & $0.005^{\mathrm{a}}$ & $0.615-0.933$ \\
\hline CRP & 0.669 & 0.094 & 0.080 & $0.484-0.853$ \\
\hline CEA & 0.529 & 0.103 & 0.768 & $0.326-0.731$ \\
\hline CA19-9 & 0.570 & 0.089 & 0.467 & $0.396-0.744$ \\
\hline
\end{tabular}

${ }^{a} \mathrm{P}<0.01$ vs. contol patients. AUC, area under receiver operating characteristic curve; ANGPTL2, angiopoietin-like protein 2; CRP, C-reactive protein; CEA, carcinoembryonic antigen; CA19-9, carbohydrate antigen 19-9.

\section{Discussion}

To the best of our knowledge, the present study was the first to investigate ANGPTL2 expression in gastric cancer cell lines. It was revealed that the mRNA and protein expression levels of ANGPTL2 were higher in HGC-27 cells than those in MKN7 cells. Furthermore, the expression levels of eight angiogenic factors (G-CSF, PECAM-1, HGF, VEGF, leptin, PDGF-BB, angiopoietin-2 and follistatin) that were potential biomarkers for gastric cancer were investigated. Of these potential biomarkers, VEGF expression was confirmed in both cell lines. VEGF protein mediates angiogenesis associated with tumor growth and is a predictive biomarker for tumor progression in certain types of cancer $(13,14)$. The results of the present study verified that VEGF was a biomarker for gastric cancer. The results further indicated that ANGPTL2 may also be a biomarker for gastric cancer.

The potential of ANGPTL2 as a novel biomarker for gastric cancer was evaluated by measuring ANGPTL2 concentration in the serum of gastric cancer patients. It was revealed that the ANGPTL2 concentration in gastric cancer patients was higher than that of healthy controls. According to the ROC analysis, ANGPTL2 demonstrated greater diagnostic ability than the classic biomarkers (CRP, CEA and CA19-9).

It was previously reported that ANGPTL2 was closely associated with adiposity and inflammation $(15,16)$. Therefore, the present study examined correlations between the serum ANGPTL2 levels, serum CRP levels and the BMI. The serum ANGPTL2 levels of the gastric cancer patients and healthy controls were not correlated with the serum CRP concentration and the BMI (Table II). These findings suggested that ANGPTL2 was a specific factor for gastric cancer.

In conclusion, these results demonstrated that ANGPTL2 was upregulated in undifferentiated gastric cancer cells and patients, which indicated that ANGPTL2 was a clinically relevant biomarker for gastric cancer.

\section{Acknowledgements}

The present study was supported in part by the Division of Gene Research, Kagoshima University and the Divisions of Gastrointestinal Surgery and Clinical Laboratory, Nanpuh Hospital (Kagoshima, Japan). 


\section{References}

1. Loei H, Tan HT, Lim TK, Lim KH, So JB, Yeoh KG and Chung MC: Mining the gastric cancer secretome: identification of GRN as a potential diagnostic marker for early gastric cancer. J Proteome Res 11: 1759-1172, 2012.

2. Yu X, Luo L, Wu Y, Yu X, Liu Y, Yu X, Zhao X, Zhang X, Cui L, Ye G, et al: Gastric juice miR-129 as a potential biomarker for screening gastric cancer. Med Oncol 30: 365, 2013.

3. Tan YK and Fielding JW: Early diagnosis of early gastric cancer. Eur J Gastroenterol Hepatol 18: 821-829, 2006.

4. Li C, Li JF, Cai Q, Qiu QQ, Yan M, Liu BY and Zhu ZG: MiRNA-199a-3p: A potential circulating diagnostic biomarker for early gastric cancer. J Surg Oncol 108: 89-92, 2013.

5. Carpelan-Holmström M, Louhimo J, Stenman UH, Alfthan $H$ and Haglund C: CEA, CA 19-9 and CA 72-4 improve the diagnostic accuracy in gastrointestinal cancers. Anticancer Res 22: 2311-2316, 2002.

6. Lukaszewicz-Zając M, Mroczko B, Gryko M, Kędra B and Szmitkowski M: Comparison between clinical significance of serum proinflammatory proteins (IL-6 and CRP) and classic tumor markers (CEA and CA 19-9) in gastric cancer. Clin Exp Med 11: 89-96, 2011.

7. Kikuchi R, Tsuda H, Kozaki K, Kanai Y, Kasamatsu T, Sengoku K, Hirohashi S, Inazawa J and Imoto I: Frequent inactivation of a putative tumor suppressor, angiopoietin-like protein 2 , in ovarian cancer. Cancer Res 68: 5067-5075, 2008.

8. Oike Y, Yasunaga K and Suda T: Angiopoietin-related/angiopoietin-like proteins regulate angiogenesis. Int J Hematol 80 21-28, 2004.

9. Sfiligoi C, de Luca A, Cascone I, Sorbello V, Fuso L, Ponzone R, Biglia N, Audero E, Arisio R, Bussolino F, et al: Angiopoietin-2 expression in breast cancer correlates with lymph node invasion and short survival. Int J Cancer 103: 466-474, 2003.
10. Endo M, Nakano M, Kadomatsu T, Fukuhara S, Kuroda H, Mikami S, Hato T, Aoi J, Horiguchi H, Miyata K, et al: Tumor cell-derived angiopoietin-like protein ANGPTL2 is a critical driver of metastasis. Cancer Res 72: 1784-1794, 2012.

11. Jass JR and Sobin LH: Histological Typing of Intestinal Tumours. 2nd edition, Springer-Verlag, Berlin-Heidelberg-New York, 1989.

12. Okada T, Tsukano H, Endo M, Tabata M, Miyata K, Kadomatsu T, Miyashita K, Semba K, Nakamura E, Tsukano M, et al: Synoviocyte-derived angiopoietin-like protein 2 contributes to synovial chronic inflammation in rheumatoid arthritis. Am J Pathol 176: 2309-2319, 2010.

13. Presta LG, Chen H, O'Connor SJ, Chisholm V, Meng YG, Krummen L, Winkler M and Ferrara N: Humanization of an anti-vascular endothelial growth factor monoclonal antibody for the therapy of solid tumors and other disorders. Cancer Res 57: 4593-4599, 1997.

14. Wang Q, Diao X, Sun J and Chen Z: Stromal cell-derived factor-1 and vascular endothelial growth factor as biomarkers for lymph node metastasis and poor cancer-specific survival in prostate cancer patients after radical prostatectomy. Urol Oncol 31: 312-317, 2013

15. Tabata M, Kadomatsu T, Fukuhara S, Miyata K, Ito Y, Endo M, Urano T, Zhu HJ, Tsukano H, Tazume H, et al: Angiopoietin-like protein 2 promotes chronic adipose tissue inflammation and obesity-related systemic insulin resistance. Cell Metab 10: 178-188, 2009.

16. Doi Y, Ninomiya T, Hirakawa Y, Takahashi O, Mukai N, Hata J, Iwase M, Kitazono T, Oike Y and Kiyohara Y: Angiopoietin-like protein 2 and risk of type 2 diabetes in a general Japanese population: the Hisayama study. Diabetes Care 36: 98-100, 2013. 